Les ANNALES Les Annales de droit

DE DROIT

11 | 2017

Varia

\title{
Le monopole persistant des huissiers de justice en
} France

The continued monopoly of bailiffs in France

Nawel Belmanaa

\section{OpenEdition}

1 Journals

Édition électronique

URL : http://journals.openedition.org/add/485

DOI : 10.4000/add.485

ISSN : 2606-1988

Éditeur

Presses universitaires de Rouen et du Havre

Édition imprimée

Date de publication : 16 octobre 2017

Pagination : 09-19

ISBN : 979-10-240-0775-5

ISSN : 1955-0855

Référence électronique

Nawel Belmanaa, « Le monopole persistant des huissiers de justice en France », Les Annales de droit [En ligne], 11 | 2017, mis en ligne le 16 octobre 2018, consulté le 19 avril 2019. URL : http://

journals.openedition.org/add/485; DOI : 10.4000/add.485 


\section{Le monopole persistant des huissiers de justice en France}

Nawel BelmanaA

Promulguée le 6 août 2015, la loi $n^{0}$ 2015-990 pour la croissance, l'activité et l'égalité des chances économiques - dite "Macron » est censée libérer les professions juridiques règlementées. Cependant, les huissiers de justice conserveront leur monopole. On peut alors se demander si les motifs qui ont présidé à l'octroi de ce monopole sont encore d'actualité.

Le principe de liberté du commerce et de l'industrie constitue un des piliers du droit commercial français ${ }^{1}$. Ce principe implique, d'une part, la liberté d'entreprendre et, d'autre part, la liberté d'exploiter. Cependant, au fil de son existence, ce principe s'est vu opposer certaines limites. Il existe des restrictions liées, notamment, à une incompatibilité des professions avec l'exercice du commerce. Il s'agit des activités participant à l'exercice de l'autorité publique.

Ainsi, il est formellement interdit d'exercer une activité commerciale lorsque l'on a la qualité de fonctionnaire. Il en va de même pour certaines professions libérales ou encore pour les officiers ministériels. Effectivement, les magistrats, les huissiers de justice, les notaires, les commissaires-priseurs concourent tous au fonctionnement de l'administration judiciaire ou du service public de la justice. À ce titre, ces professions peuvent bénéficier d'entrave au principe de liberté du commerce et de l'industrie et ainsi bénéficier d'un certain monopole d'État.

Mais avec l'émergence des idées économiques libérales au plan national et européen, la critique des monopoles se fait jour : ils apparaissent comme abusifs et nuisibles aux consommateurs et à la circulation des biens et des services. Dans un contexte de libéralisation économique et

1. Principe posé par l'article 7 de la loi des 2-17 mars 1791 (loi d'Allarde). Le Conseil d'État consacre ce principe comme une liberté publique par l'arrêt CE, 28 oct. 1960 , req. $\mathrm{n}^{\mathrm{o}} 48293$, Martial de Laboulaye. Le principe a valeur constitutionnelle par la décision du Cons. const., 16 janv. 1982, $\mathrm{n}^{\circ} 81-132$ DC, relative aux lois de nationalisation consacrant la liberté d'entreprendre, Rec. 18. 
d'influence du droit communautaire, les limites à la liberté du commerce et de l'industrie sont-elles en train de disparaitre? L'influence grandissante du droit communautaire sur le droit national peut pousser à une redéfinition du principe français de liberté du commerce et de l'industrie.

La question de l'ouverture des professions réglementées est d'actualité depuis le début de la $V^{\mathrm{e}}$ République avec le rapport Rueff-Armand ${ }^{2}$. Selon ce rapport, parmi les obstacles à l'expansion économique, on trouve les professions réglementées. Ouvrir ces professions à la concurrence permettrait de générer de la croissance, de baisser les prix et d'augmenter les revenus.

En novembre 2014, le député Richard Ferrand a remis au garde des Sceaux, ministre de la Justice, un rapport proposant diverses mesures de modernisation des professions réglementées et, notamment, des conditions d'installation plus souples, des tarifs plus transparents et orientés vers les coûts. La synthèse du rapport Ferrand dresse même un constat alarmant: « Ne pas moderniser et ne pas adapter les professions dites réglementées serait les condamner ${ }^{3}$.»

Le rapport Darrois qui constitue la base de la loi $\mathrm{n}^{0} 2011-331 \mathrm{du}$ 28 mars 2011, sur la modernisation des professions judiciaires ou juridiques et certaines professions réglementées préconise pour assurer une cohérence au sein des professions de l'exécution, de rapprocher, voire de fusionner les professions d'huissier de justice, de commissaire-priseur judiciaire et de mandataire judiciaire afin de «mutualiser des moyens sur des domaines d'activité très similaires, voire concurrentiels ${ }^{4} »$. En définissant des règles permettant de renforcer les professions du droit pour mieux répondre aux besoins des usagers, la loi $\mathrm{n}^{\mathrm{o}} 2011-331 \mathrm{du} 28$ mars 2011 a dégagé des principes qui gardent aujourd'hui toute leur actualité 5 .

C'est dans ce même esprit que la loi no 2015-990 du 6 août 2015 pour la croissance, l'activité et l'égalité des chances économiques a été promulguée. Le volet de la loi sur la libéralisation des professions réglementées vise à promouvoir une réforme profonde des professions

2. Voir Jacques Rueff et Louis Armand, «Rapport sur les obstacles à l'expansion économique», Paris, La Documentation française, p. 32.

3. Voir le rapport de Richard Ferrand, Laure Deltour-Becq et Amaury Dumay, «Professions réglementées. Pour une nouvelle jeunesse», Paris, La Documentation française, novembre 2014, p. 3.

4. Voir le rapport de Jean-Michel Darrois sur les professions du droit, «Vers une grande profession du droit », Paris, La Documentation française, avril 2009.

5. Pour une présentation détaillée, voir Olivier Salati, «Présentation de la loi du 28 mars 2011 de modernisation des professions judiciaires ou juridiques et certaines professions réglementées », Dr. et proc. 2011, p. 110-116. 
du droit destinée tout à la fois à favoriser la concurrence et leur compétitivité interne et internationale, et à améliorer la qualité des services rendus au profit de tous les usagers du droit. Cependant, malgré la promulgation de la loi «Macron", les huissiers de justice conserveront leur monopole. Or, le monopole de l'huissier de justice est considéré, aujourd'hui, comme empiétant sur la libre concurrence ce qui peut constituer un frein à la compétitivité de la profession. Mais peut-on concilier la compétitivité économique avec l'exercice du service public de la justice par un huissier de justice en France?

Pour répondre à cette question, il convient de rappeler que des motifs ont justifié l'octroi d'un monopole aux huissiers de justice en France, particulièrement pour garantir le service de la justice (1). Cependant, certaines attributions liées au monopole de l'huissier de justice peuvent être contestées (2) et constituer un frein à l'adaptation de la profession face aux exigences actuelles d'ouverture du «marché du droit» (3).

\section{Le monopole de l'huissier de justice garant du service public de la justice}

Les officiers d'état civil, les notaires, les huissiers de justice sont des officiers publics, qu'ils soient élus par leurs concitoyens pour certains, nommés par le gouvernement après enquête de moralité et examen professionnel pour d'autres. L'officier public exerce une mission d'intérêt général qui lui est conférée par le législateur. Cette mission d'intérêt général, participant directement au service public de la justice, est assortie d'un monopole légal qui permet un exercice serein de leurs fonctions.

C'est ainsi que les notaires ont le monopole de la rédaction des actes authentiques pour les particuliers et sont soumis à une obligation de résultat dans leur rédaction. En vertu de l'article 1319 du Code civil, les notaires sont «établis à recevoir tous les actes et contrats auxquels les parties doivent ou veulent faire donner un caractère d'authenticité attachée aux actes de l'autorité publique». Cette disposition prévient tout vice de consentement des parties et garantit la conformité de l'acte aux lois et aux règlements. Le monopole de la rédaction des actes authentiques des notaires est donc nécessaire à la protection de l'ordre public juridique.

Les huissiers de justice occupent également une place importante dans le système juridique français et créent de nombreux emplois ${ }^{6}$.

6. En 2014, la France comptait plus de 3142 huissiers de justice d'après l'observatoire économique de la Chambre nationale des huissiers de justice (CNHJ). 
L'huissier de justice est un officier public et ministériel exerçant une profession libérale réglementée. Bénéficiant du monopole de l'exécution des décisions de justice et de la délivrance des actes, il peut constater de manière impartiale les faits qui conduisent à la preuve ${ }^{7}$. Les huissiers de justice œuvrent donc dans l'intérêt d'une bonne administration de la justice, ce qui fait d'eux un élément essentiel de l'État de droit.

L'article 55 du traité de Rome préserve les huissiers de la concurrence issue de la libre installation des professionnels dans l'espace européen pour les activités relevant de leur monopole, en raison de la délégation de puissance publique dont ils bénéficient.

La profession attache aujourd'hui une importance capitale à ce monopole. L'actualité des mois précédents en est la preuve: une simple discussion du gouvernement à propos de la suppression de celui-ci a fait descendre des milliers d'huissiers de justice dans la rue, qui ont manifesté leur opposition à la loi « Macron $^{8}$ ». Pour certains, les répercussions d'une telle orientation entraîneraient une vague de licenciement importante. De plus, la liberté d'installation permettrait aux huissiers de justice de s'établir dans des zones plus attractives économiquement au détriment d'autres, créant ainsi des « déserts juridiques». Ceci dit, il ne faut pas oublier que le monopole des huissiers de justice est une véritable restriction au droit de la concurrence ${ }^{9}$.

Parmi les activités monopolistiques de l'huissier de justice, la signification des actes judiciaires dont le monopole était contesté n'est finalement pas comprise dans la réforme «Macron». Au nom de l'État, les huissiers de justice ont pour fonction essentielle d'assurer le respect du contradictoire par la signification des actes. Certains actes doivent être signifiés par huissiers de justice afin de garantir une bonne information à même de préserver les droits de la personne visée par l'acte. Ces actes font d'ailleurs souvent courir des délais de recours. Ainsi, la signification d'acte par huissier de justice garantit le contenu de l'acte et le respect de la procédure.

7. La délivrance des actes est un fondement consacré par l'art. L. 122-1 du Code des procédures civiles d'exécution (anc. L. 1991, art. 18), ainsi que par l'ordonnance $\mathrm{n}^{\mathrm{o}}$ 45-2592, 2 novembre 1945 , art. $1^{\mathrm{er}}$, relative au statut des huissiers de justice.

8. Lors de la présentation du projet de loi pour la croissance et l'activité le 10 décembre 2014 en Conseil des ministres, les six professions réglementées juridiques ont manifesté dans la rue afin d'exprimer leur rejet du texte.

9. Voir les dispositions modifiées de l'ordonnance du $1^{\mathrm{er}}$ décembre 1986 relative à la liberté des prix et à la concurrence, codifiées aux articles L. 442-1 et suivants du Code de commerce, ainsi qu'aux articles 81 et 82 du traité CE. 
L'acte d'huissier est un acte solennel qui doit respecter certaines formes obligatoires à peine de nullité, lui donnant une force probante incontestable ${ }^{10}$. En vertu de l'article 1317 du Code civil, «l'acte authentique est celui qui a été reçu par officiers publics ayant le droit d'instrumenter dans le lieu où l'acte a été rédigé, et avec les solennités requises ». En sa qualité d'officier public, l'huissier de justice, tout comme le notaire, confère à ses actes un caractère authentique. L'authenticité des actes est fondée sur la confiance qu'inspirent les officiers publics. La véracité des actes est garantie par le fait qu'ils n'ont jamais d'intérêt personnel aux actes qu'ils reçoivent, puisqu'il leur est interdit d'instrumenter pour eux-mêmes ou les membres de leur famille. De même, ils s'exposent à de graves sanctions pénales et civiles en cas de faux. En vertu de l'article 1319 du Code civil, «l'acte authentique fait pleine foi de la convention qu'il renferme». Sa force probante est supérieure à celle qui est attachée à l'acte sous seing privé. L'acte authentique fait foi jusqu'à l'inscription de faux de sa réalité et des constatations de l'officier public ${ }^{11}$. Toutes les mentions contenues dans l'acte d'huissier ne revêtent pas la qualité d'authenticité. La force probante ne s'attache qu'aux faits et aux formalités accomplis par l'huissier de justice comme le lieu, la date de remise... La date figure parmi les mentions obligatoires au titre de l'article 648 du Code de procédure civile. La signification par un huissier de justice permet donc de prouver la date de la remise de l'acte au destinataire.

Force est de constater que la signification faite par un huissier de justice assure une certaine sécurité juridique : l'acte d'huissier de justice est remis en main propre, y compris sur le lieu de travail, et à défaut à une personne présente au domicile. Si personne ne veut ou ne peut recevoir l'acte, il est déposé et conservé pendant trois mois à l'étude de l'huissier de justice qui aura vérifié l'adresse du destinataire de l'acte ${ }^{12}$. La signification par un huissier de justice peut garantir la délivrance effective de l'acte et informe le destinataire de son contenu. En tant qu'auxiliaire de justice de proximité, l'huissier ne doit pas se contenter de remettre l'acte, mais doit également informer et expliquer ses droits au destinataire de l'acte.

En ce sens, on peut considérer que la signification des actes par un huissier de justice est une activité nécessaire à l'accomplissement d'une mission de service public de la justice, qui conduit à la non-application

10. Art. 648 et 649 du Code de procédure civile.

11. Art. 1319 du Code civil.

12. Art. 655 du Code de procédure civile. 
des règles de la liberté du commerce et de l'industrie et du droit de la concurrence.

\section{Un monopole parfois contestable}

Pourtant, même si l'huissier de justice a le monopole de la signification des actes authentiques, c'est généralement un clerc significateur qui mène à bien cette opération ${ }^{13}$.

Dans les grandes agglomérations, la signification des actes est même organisée en «bureau commun». Des clercs significateurs collectent les actes de procédure contresignés par l'huissier de justice, avant de partir les signifier eux-mêmes pour le compte des huissiers titulaires d'une étude. Ces groupements sont régis par un décret du 29 février 1956, afin de faciliter l'exécution de leur travail matériel et réduire leurs frais d'exploitation ${ }^{14}$. Les huissiers de justice ont donc la possibilité, moyennant paiement, de déléguer la signification de leurs actes. Ainsi, dans un souci d'efficacité et de rentabilité, la signification de nombreux actes se fait par l'intermédiaire des clercs significateurs.

La signification des actes ne relève donc pas spécifiquement du domaine de l'huissier de justice. Dans ce contexte, l'hypothèse de l'ouverture de cette activité à d'autres professions telles que les postiers ne paraît pas inconcevable. Toute restriction doit être justifiée par une raison impérieuse d'intérêt général et il n'apparaît pas de raison valable d'exclure d'autres professions de la signification des actes.

Les huissiers de justice ne seraient-ils pas seulement guidés par une inquiétude infondée de la libéralisation de la profession? Ou alors, existe-t-il une réelle menace pour la profession?

Face à la baisse du nombre d'actes de signification due à une concurrence accrue des services contentieux des maisons de crédit et autres sociétés de recouvrement, la signification des actes ne pourrait à elle seule permettre de subsister sans le développement d'autres activités. Au vu de l'état actuel du marché, s'attacher à ce seul monopole n'est donc pas judicieux. La signification des actes par voie d'huissier de justice devrait

13. Sur le domaine des compétences partagées entre l'huissier de justice et le clerc significateur, voir la loi du 27 décembre 1923 sur le statut du clerc assermenté et le commentaire d'Olivier Salati sur «l'exécution forcée, domaine réservé de l'huissier de justice", Code des procédures civiles d'exécution commenté, Paris, Dalloz, 2015, p. 102-103.

14. D. $\mathrm{n}^{\mathrm{O}}$ 56-222, 29 févr. 1956 sur l'application de l'ordonnance du 2 novembre 1945 relative au statut des huissiers de justice. 
être limitée aux actes dont la gravité et les effets justifient qu'ils soient confiés à un officier public ministériel.

Libérer la profession du monopole de la signification des actes n'est peut-être qu'une question de lucidité et de courage politique. En effet, pour libérer "le marché de la signification des actes», le gouvernement devra renoncer à d'importantes recettes fiscales liées à la taxe forfaitaire des actes de significations en constante augmentation et subir de nombreuses pressions de la part de la profession ${ }^{15}$.

Par ailleurs, le gouvernement soutient la nécessité de libérer les professions du droit afin de répondre au mieux à la demande, ce qui implique une liberté des prix, une concurrence et une liberté d'installation. À compter du $1^{\text {er }}$ février 2016, les huissiers de justice, les notaires et commissaires-priseurs peuvent librement s'installer dans les zones où l'implantation d'offices apparaît nécessaire pour renforcer l'offre de service. Ces zones sont déterminées par le ministre de la Justice: une "carte des régions » est établie par le gouvernement sur proposition de l'Autorité de la concurrence pour l'implantation des offices de notaires, huissiers de justice et commissaires-priseurs judiciaires. La liberté d'installation reste donc limitée, puisque c'est l'État qui règle l'organisation territoriale de ces professions juridiques. Ce décret du 26 février $2016^{16}$ va juste permettre d'augmenter de quelques études le numerus clausus déjà en place. On est donc loin d'instaurer la liberté d'installation pour les notaires et les huissiers de justice ${ }^{17}$.

De toute manière, face aux exigences actuelles du marché, les huissiers de justice ont tout intérêt à anticiper l'évolution de la profession. Le fait que la profession d'huissier de justice bénéficie d'un monopole légal en France ne signifie pas qu'elle échappe à toute menace.

\section{Le monopole de l'huissier de justice face aux exigences actuelles d'ouverture du «marché du droit »}

Les huissiers de justice exercent de manière concurrentielle dans de nombreux domaines: recouvrement amiable de créance, aide à la

15. CGI, art. 302 bis $\mathrm{Y}$ : «Les actes des huissiers de justice sont soumis à une taxe forfaitaire de 13,04 € pour les actes accomplis à compter du ${ }^{\mathrm{er}}$ janvier 2016 et $14,89 €$ pour les actes accomplis à compter du $1{ }^{\mathrm{er}}$ janvier 2017. »

16. D. $n^{0}$ 2016-216, 26 févr. 2016 relatif à l'établissement de la carte instituée au I de l'article 52 de la loi $n^{\circ}$ 2015-990, 6 août 2015 pour la croissance, l'activité et l'égalité des chances économiques.

17. Voir l'article de Jean-Yves Naudet sur «Loi Macron : libéralisation ou régulation? », Contrepoints, 24 décembre 2014. 
rédaction des actes sous seing privé, ventes aux enchères publiques, consultations juridiques, administration d'immeubles... Suite à la promulgation le 6 août 2015 de la loi « Macron", ils pourront même exercer le recouvrement des petites créances impayées de nature contractuelle sans avoir recours à une action en justice ${ }^{18}$.

Aujourd'hui plus que jamais l'huissier de justice doit être un véritable chef d'entreprise capable d'investir ces différentes activités afin de pouvoir faire face aux nouvelles exigences du marché et se diriger rapidement vers les secteurs les plus porteurs. L'huissier de justice de demain sera un "homme-orchestre" assurant de multiples fonctions qui seraient fragmentées en plusieurs services (administratif, technique, financier, ressources humaines...)

L'environnement économique dans lequel évoluent les huissiers de justice connaît une profonde transformation et les soumet à une concurrence acharnée. Ainsi, même si les tarifs des huissiers de justice sont strictement réglementés dans le cadre de leurs activités monopolistiques et qu'il existe un numerus clausus qui limite le nombre des études d'huissiers en France, la concurrence au sein de cette profession ne cesse de se développer.

Celle-ci est très variée, elle peut provenir des huissiers eux-mêmes ou des sociétés de recouvrement.

Effectivement, la compétence territoriale des huissiers de justice pour la signification des actes et l'exécution des décisions de justice a pour base le ressort du tribunal de grande instance de la résidence de l'huissier de justice ${ }^{19}$. Mais à la suite de la promulgation de la loi "Macron", à compter du $1{ }^{\text {er }}$ janvier 2017, la compétence territoriale des huissiers de justice pour la signification des actes et l'exécution des décisions de justice s'exercera dans le ressort de la cour d'appel de la résidence professionnelle de l'huissier ${ }^{20}$. Autrement dit, la compétence des huissiers de justice ne sera plus départementale, mais régionale. Cette récente réforme permettra d'étendre le champ de compétence des huissiers de justice et de créer ainsi, une hausse de compétitivité puisque le client va voir son choix augmenter. Cette concurrence conduirait les différentes études d'huissiers à vouloir fournir des prestations de qualité

18. L. $\mathrm{n}^{\mathrm{o}}$ 2015-990, art. 208: "Les huissiers de justice pourront délivrer eux-mêmes un titre exécutoire dès l'accord du créancier et du débiteur sur le montant et les modalités de paiement de la créance».

19. Voir D. $\mathrm{n}^{\mathrm{0}}$ 56-222, 29 févr. 1956, art. 5.

20. Voir Ord. $\mathrm{n}^{\mathrm{o}}$ 45-2592, 2 nov. 1945, art. 3, al. $1^{\mathrm{er}}$, modif. par L. Macron, art. 54. 
toujours supérieure. Le risque serait la disparition des petites études qui ne feraient plus le poids au niveau régional contre les grosses structures.

De plus, les huissiers de justice comme les sociétés de recouvrement peuvent être choisis par les créanciers pour procéder au recouvrement amiable des créances. Cependant, les petites études d'huissiers sont souvent mal équipées pour gérer des dossiers en masse, les créanciers préfèrent alors s'adresser aux sociétés de recouvrement. Face à ces nouveaux impératifs du marché, certaines études n'ont pas d'autre alternative que de concentrer leurs structures d'exercice afin d'optimiser leurs investissements faisant diminuer ainsi le nombre d'études d'huissiers en France $^{21}$.

C'est pourquoi, l'huissier de justice ne doit pas subir cette concurrence passivement, mais s'adapter à la situation, au risque de subir un jour la concurrence sans aucun ménagement. Ainsi, si le monopole des huissiers de justice est nécessaire à une bonne administration de la justice, il ne pourrait à lui seul permettre aux huissiers de justice de subsister sans le développement d'autres activités. A contrario, selon l'Autorité de la concurrence ${ }^{22}:$ «l'accroissement de la concurrence sur le territoire peut réduire les coûts des actes et renforcer la qualité et l'efficacité des services ». La fin du monopole de la signification des actes aurait pu permettre de favoriser la compétitivité interne et internationale de la profession des huissiers de justice et améliorer la qualité des services rendus au profit de tous les usagers du droit. Face à la concurrence, les huissiers de justice n'auront pas d'autres choix que d'améliorer autant que faire se peut la qualité de leurs services et de s'adapter à la demande, notamment par la signification par voie électronique ${ }^{23}$ et la mise en place d'échange des données informatisées ${ }^{24}$.

Nous pouvons donc regretter que la loi $n^{0} 2015-990$ n'ait pas reformulé le périmètre de ce monopole tout en gardant à l'esprit les dangers d'une trop grande libéralisation de la profession. Car une trop grande concurrence peut, à terme, inciter les huissiers de justice à privilégier

21. Selon le rapport de l'inspection générale des finances sur les professions réglementées datant d'avril 2013, on note une diminution de $10 \%$ d'études d'huissiers dans la période 2005-2012.

22. Sollicitée par le gouvernement, l'Autorité de la concurrence encourage, dans un avis $\mathrm{n}^{0}$ 15-A-02 du 9 janvier 2015, l'ouverture de certaines professions juridiques réglementées.

23. Voir D. $\mathrm{n}^{\mathrm{o}}$ 2012-366, 15 mars 2012 relatif à la signification des actes d'huissier de justice par voie électronique et aux notifications internationales.

24. L'EDI (échange des données informatisées) permet d'accélérer la transmission informatique des documents nécessaires au recouvrement judiciaire des créances. 
encore plus la recherche du profit au détriment des particuliers, mettant ainsi en péril leur tâche publique. Il ne faut pas oublier qu'un huissier de justice doit avant tout accomplir ses missions dans l'intérêt général et avec le souci du justiciable en respectant les règles déontologiques et éthiques inhérentes à la profession.

Un point clé est de savoir quel est le devenir des activités monopolistiques des huissiers de justice en France face à la politique de libéralisation des professions réglementées menée par les autorités européennes.

Effectivement, la directive du 12 décembre 2006 relative aux services dans le marché intérieur, plus communément appelée «directive services » ou "directive Bolkestein », réaffirme la libre prestation de service au sein des états membres de l'Union, inscrite dès 1957 dans le traité de Rome $^{25}$. Alors que les professions réglementées, en particulier dans les domaines du droit ont longtemps échappé à l'application de ce principe, la direction générale de la concurrence de la Commission européenne enjoint les États membres à les rendre conformes aux règles de la concurrence européenne ${ }^{26}$. Dans ce cadre, avocats, notaires, pharmaciens, comptables, mais aussi architectes et ingénieurs sont invités à éliminer les règles en vigueur dans leur profession occasionnant une atteinte à la concurrence dans leur secteur d'activité. Lors de la transposition en droit français de la directive, les huissiers de justice, ainsi que les notaires, ont été exclus du champ d'application de cette dernière, grâce à leur statut d'officier public et ministériel.

Ainsi, bien que la transposition en droit français de la «directive Bolkenstein » exclue de son champ d'application les huissiers de justice, son adoption démontre le risque de déréglementation auquel est soumise aujourd'hui la profession d'huissier.

Plus récemment encore, le 30 mai 2012, la Commission européenne a adressé une recommandation du Conseil concernant le programme national de réforme de la France pour 2012 et portant avis du Conseil sur le programme de stabilité de la France pour la période 2012-2016, dans laquelle elle mentionne au point $\mathrm{n}^{\mathrm{o}} 15$ la nécessité de réformer le statut de différentes professions juridiques. La déréglementation est présentée comme une composante essentielle visant à favoriser la compétitivité du «marché du droit».

25. Voir dir. $\mathrm{n}^{\mathrm{0}}$ 2006/123/CE, 12 déc. 2006.

26. Voir le rapport sur la concurrence dans le secteur des professions libérales, Bruxelles, 9 février 2004, COM (2004) et suivi du rapport sur la concurrence dans le secteur des professions libérales, Bruxelles, 5 septembre 2005, COM (2005). 
Selon la Commission européenne, un allègement des restrictions à la concurrence sur le marché du droit permettrait de libérer le potentiel de croissance du marché de services en Europe grâce à l'élimination des obstacles juridiques et administratifs de nature à freiner les échanges et la circulation des services.

Si depuis plus d'un demi-siècle d'études et de projets de réforme, que ce soit au plan national ou au plan européen, le statut particulier des huissiers de justice garde ses particularités, on peut se demander pendant combien de temps encore cela peut perdurer. Les études d'huissiers de justice sont en effet confrontées à d'importantes évolutions d'ordre réglementaire, économique, stratégique et technologique qu'il convient d'anticiper au risque de mettre en péril la profession d'huissier de justice.

Doctorante en droit à Aix-Marseille Université, sous la direction d'Olivier Salati, maître de conférences à l'université d'Aix-Marseille III 